\title{
Atención al usuario y comunicación en los portales web de salud autonómicos en España
}

\author{
Xosé María Mahou Lago \\ Universidad de Vigo \\ xmahou@uvigo.es
}

Ramón Bouzas Lorenzo

Universidad de Santiago de Compostela

ramon.bouzas@usc.es

\begin{abstract}
Resumen
Se presentan los resultados de un estudio llevado a cabo durante 2011 mediante las técnicas de test heurístico y e-usuario misterioso, sobre la variedad y eficacia de los dispositivos de comunicación vía web puestos por las administraciones autonómicas españolas al servicio de los ciudadanos dentro del área de salud. Los resultados demuestran, en general, que las webs gestionadas por las administraciones autonómicas ofrecen un potencial tecnológico muy superior al que exige la reducida gama de prestaciones que en la actualidad se ofrecen a los usuarios de los servicios de salud. Asimismo, los datos recabados ponen en evidencia graves deficiencias en la oferta y la gestión de los dispositivos de comunicación con los usuarios y, en especial, los que estimulan la captación de feedback a través de la participación activa de los ciudadanos, componiendo un panorama todavía alejado de un empleo aceptable de un modelo de e-salud 2.o.
\end{abstract}

Palabras clave

e-Gobierno, portal web, políticas públicas, comunicación, salud.

\section{User support and communication in health web portals of the Spanish Autonomous Administrations}

\begin{abstract}
Based on the use of heuristic test and e-mystery user techniques, the article presents the results of a study conducted in 2011 about the variety and effectiveness of web-based communication devices laid by the Spanish regional governments in order to provide health services to citizens. The results show that the websites operated by regional governments offer much higher technological capabilities than those required by the narrow range of benefits currently offered to users of health services. Also, data collected highlights serious shortcomings in the supply and management of communication devices, especially those that encourage citizens' feed back through their active participation. Results depict a panorama still far from a reasonable use of an e-health 2.0 model.
\end{abstract}

Keywords

e-Government, web portal, public policy, communication, health. 


\section{INTRODUCCIÓN}

Los progresos logrados en la interacción entre los ciudadanos y las Administraciones a través de Internet (Comisión Europea, 2002; OCDE, 2005; UNDESA, 2010) han contribuido a transformar el modo de entender las relaciones entre los diversos actores involucrados en el proceso de implementación de políticas públicas y, en concreto, en la provisión de servicios públicos. Se produce lo que se ha denominado el giro al usuario, metáfora empleada para poner de relieve la necesidad de que las Administraciones se preocupen de las demandas e intereses de aquellos a los que sirven (Schedler y Proeller, 2003).

La materialización del propósito de servir a los ciudadanos con mayor eficiencia, en un entorno de e-gobierno 2.0, puede encontrarse en la transición desde una oferta de sitios web institucionales centrados en la provisión de información sobre la propia organización, y su gama de servicios públicos, a la activación de portales sectoriales.

El portal, vía de entrada única e integrada que facilita a usuarios externos e internos el acceso a recursos y servicios oficiales relacionados con un área competencial, representa un avance en el enfoque de provisión de e-servicios y aspira a convertirse en una herramienta de gran potencial en la implementación de políticas públicas.

El presente trabajo', que toma como objeto de análisis los portales de los servicios públicos autonómicos, responde al objetivo de informar acerca del nivel de desarrollo alcanzado por los dispositivos de comunicación web en el marco de la provisión de información y servicios públicos relacionados con la salud, una de las áreas en las que con mayor visibilidad y utilidad social se han producido avances en la oferta de servicios electrónicos y mecanismos de participación (Comisión Europea, 2004; Andreassen et al., 2007; Eysenbach, 2008; OMS/WHO, 2011).

\section{MÉTODO}

El método empleado para el análisis de la capacidad de comunicación de las webs autonómicas de salud se inspiró en los estudios que durante los últimos veinte años se han hecho sobre usabilidad, y tomó como unidad de análisis la web de salud de cada administración autonómica, cuya arquitectura arquetípica presenta hasta cuatro puntos de acceso para el usuario: el portal principal de la administración autonómica, el portal del departamento sectorial (consejería), el portal del servicio autonómico de salud y el portal temático (especializado en temas salud) gestionado por la propia administración (tabla 1).

$1 \quad$ Los datos que se exponen son el resultado de una parte del proyecto de investigación Barreras al Gobierno Electrónico. El Empleo de las TIC en la Política Sanitaria Autonómica. Un Enfoque desde las Relaciones Intergubernamentales (CSO2009-09169). 
TABLA 1

Disponibilidad de vías de acceso a las webs de salud gestionadas por las administraciones autonómicas $2011^{2}$

\begin{tabular}{|c|c|c|c|c|}
\hline & $\begin{array}{c}\text { Portal } \\
\text { Administración } \\
\text { autonómica }\end{array}$ & $\begin{array}{c}\text { Departamento } \\
\text { de Salud }\end{array}$ & $\begin{array}{c}\text { Servicio } \\
\text { de Salud } \\
\text { autonómico }\end{array}$ & $\begin{array}{c}\text { Portal } \\
\text { de Salud } \\
\text { autonómico }\end{array}$ \\
\hline Andalucía & Sí & $\begin{array}{l}\text { Sí (compartida } \\
\text { con portal) }\end{array}$ & Sí & $\begin{array}{l}\text { Sí (compartida } \\
\text { con } \\
\text { departamento) }\end{array}$ \\
\hline Aragón & Sí & Sí & Sí & Sí \\
\hline Asturias & Sí & Sí & \multicolumn{2}{|c|}{ Sí (compartida) } \\
\hline Baleares & Sí & \multicolumn{2}{|c|}{ Si (compartida) } & Sí \\
\hline Canarias & Sí & Sí & Sí & No \\
\hline Cantabria & Sí & No & Sí & No \\
\hline Castilla y León & Sí & Sí & \multicolumn{2}{|c|}{ Sí (compartida) } \\
\hline Castilla-La Mancha & Sí & Sí & Sí & No \\
\hline Cataluña & Sí & Sí & Sí & No \\
\hline Madrid & Sí & Sí & No & Sí \\
\hline Valencia & Sí & \multicolumn{2}{|c|}{ Sí (compartida) } & No \\
\hline Extremadura & Sí & Sí & No & Sí \\
\hline Galicia & Sí & \multicolumn{2}{|c|}{ Si (compartida) } & No \\
\hline La Rioja & Sí & No & No & Sí \\
\hline Navarra & Sí & Sí & Sí & Sí \\
\hline País Vasco & Sí & Sí & Sí & Sí \\
\hline Murcia & Sí & Sí & \multicolumn{2}{|c|}{ Sí (compartida) } \\
\hline
\end{tabular}

Fuente: Elaboración propia.

El procedimiento seguido se basó en la utilización de dos técnicas: examen heurístico (usabilidad técnica y comunicación) y experimento de e-usuario misterioso.

El examen heurístico o test de experto se aplicó entre el 16 de enero y el 28 de febrero de 2011 al estudio de la usabilidad técnica y al de los dispositivos de comunicación. Se trata de un método de diagnóstico a través del cual, personal cualificado analiza la página web y describe los problemas potenciales de ésta en relación con su visibilidad, diseño, eficiencia, funcionalidad y fiabilidad (Nielsen, 1994; Dumas y Redish, 1999; Kruk, 2000; Hassan y Martin, 2003).

2 La información expuesta corresponde a la oferta de webs a mes de marzo de 2011. 
El modelo de check-list para el examen heurístico de usabilidad técnica abarcó cinco parámetros: buscabilidad (la página debe resultar visible para el usuario, disponiendo de un entorno singularizado y gozando de las mejores posiciones en los motores de búsqueda 3 ); identidad (la página debe aportar suficiente información sobre la organización a la que pertenece); diseño-estilo (la interfaz del sitio debe ser amigable y atractiva para el usuario); navegabilidad (la página debe facilitar el desplazamiento del usuario y su acceso a los contenidos dentro del sistema) y seguridad (la página debe garantizar una experiencia de navegación lo más confidencial posible, la preservación, con todas las garantías legales, de los datos generados por la comunicación con el usuario, así como la protección de éste frente a un uso no autorizado de tales datos por terceros).

Teniendo en cuenta las directrices del consorcio World Wide Web $-\mathrm{W}_{3} \mathrm{C}-\mathrm{y}$ la literatura sobre usabilidad y e-gobierno, se otorgó el siguiente peso a cada uno de los cinco parámetros: buscabilidad (15\%), identidad (8\%), diseño-estilo (30\%), navegabilidad (35\%) y seguridad (12\%). Para comprender los cinco parámetros, se emplearon 41 indicadores cuyas escalas se ajustaron a la disponibilidad de cada elemento examinado, al nivel de calidad de los dispositivos y a su rendimiento.

Por otra parte, desde el punto de vista de la comunicación, el check-list, que contenía 11 indicadores, prestó atención a la oferta de dispositivos que favorecen la relación bidireccional y, en especial, la comunicación sincrónica con el usuario. El peso otorgado a cada uno de los tres parámetros examinados en relación con la comunicación (atención al usuario, 40\%; consulta, 30\% y debate, 30\%) tuvo en cuenta la importancia de un servicio al usuario lo más completo posible en el marco de la tecnología actualmente disponible.

Por su parte, el experimento de e-usuario misterioso se empleó para evaluar, desde la perspectiva de un usuario de servicios de salud, la capacidad de respuesta por parte de las administraciones (Voss, 2000; Leuenberger et. al., 2003; Matzler, et al., 2005).

A través del dispositivo de contacto proporcionado por el portal, empleando identidades falsas, se demandó, durante la segunda quincena de julio de 2011, atención sobre cinco requerimientos: información sobre un tema concreto de salud (vacunas y precauciones para un viaje al extranjero), indicaciones sobre el procedimiento a seguir en un servicio incluido en el catálogo on line (petición de cita médica), indicaciones sobre el procedimiento a seguir en un servicio off line (cumplimentación de un consentimiento informado), información sobre el acceso a datos personales que obran en poder de otra administración relacionados con el área de salud cuando el ciudadano ha trasladado su residencia a otra comunidad autónoma (historia clínica) y formulación de una sugerencia (posibilidad de solicitar cita médica a través del móvil).

3 Para el examen de la buscabilidad se consideró suficiente tomar como referencia el motor de búsqueda de Google, poseedor, según el Search Engine Barometer (2011), del 95,3 por ciento de cuota de mercado. (AT Internet, 2011). 
Los indicadores utilizados para medir la calidad de atención al ciudadano fueron: tiempo de respuesta; calidad del formato de respuesta (estructura, identificación del emisor y estilo); claridad (comprensibilidad) y calidad de la información suministrada (nivel de ajuste y de la respuesta a la demanda del usuario -excepto en la formulación de la sugerencia-, completud e información de referencia y contacto).

\section{RESULTADOS}

\section{Usabilidad}

Las prestaciones relacionadas con la usabilidad técnica son, en general, adecuadas en las webs autonómicas de salud (tabla 2).

\section{TABLA 2}

Calidad de prestaciones de usabilidad técnica ofrecidas por los portales autonómicos de salud

\begin{tabular}{|l|c|c|c|c|c|c|}
\hline & $\begin{array}{c}\text { Buscabilidad } \\
(15 \%)\end{array}$ & $\begin{array}{c}\text { Identidad } \\
(\mathbf{8} \%)\end{array}$ & $\begin{array}{c}\text { Diseño-Estilo } \\
(30 \%)\end{array}$ & $\begin{array}{c}\text { Navegabilidad } \\
(35 \%)\end{array}$ & $\begin{array}{c}\text { Seguridad } \\
(\mathbf{1 2 \%})\end{array}$ & $\begin{array}{c}\text { TOTAL } \\
(\text { Sobre 100) }\end{array}$ \\
\hline Andalucía & 4,5 & 8 & 28,4 & 24,1 & 12 & $\mathbf{7 7 , 0}$ \\
\hline Aragón & 8,2 & 6 & 18,8 & 20,8 & 4,5 & $\mathbf{5 8 , 4}$ \\
\hline Asturias & 4,5 & 8 & 26,1 & 20,8 & 7,5 & $\mathbf{6 6 , 9}$ \\
\hline Baleares & 6 & 7 & 24,9 & 19,8 & 4,5 & $\mathbf{6 2 , 3}$ \\
\hline Canarias & 9 & 7 & 17,3 & 23,6 & 7,5 & $\mathbf{6 4 , 4}$ \\
\hline Cantabria & 15 & 7 & 8,07 & 16,5 & 12 & $\mathbf{5 8 , 6}$ \\
\hline $\begin{array}{l}\text { Castilla-La } \\
\text { Mancha }\end{array}$ & 9 & 8 & 23,0 & 25,5 & 9 & $\mathbf{7 4 , 6}$ \\
\hline $\begin{array}{l}\text { Castilla y } \\
\text { León }\end{array}$ & 9 & 8 & 24,9 & 17,0 & 4,5 & $\mathbf{6 3 , 5}$ \\
\hline Cataluña & 4,5 & 7 & 28,4 & 24,5 & 4,5 & $\mathbf{6 9 , 0}$ \\
\hline Madrid & 9 & 8 & 30,0 & 23,6 & 12 & $\mathbf{8 2 , 6}$ \\
\hline Valencia & 9 & 6,5 & 17,6 & 19,8 & 12 & $\mathbf{6 4 , 0}$ \\
\hline Extremadura & 15 & 7 & 28,4 & 23,6 & 0 & $\mathbf{7 4 , 1}$ \\
\hline Galicia & 15 & 8 & 27,6 & 19,8 & 9 & $\mathbf{7 9 , 5}$ \\
\hline La Rioja & 15 & 7 & 25,3 & 27,4 & 12 & $\mathbf{8 6 , 8}$ \\
\hline Navarra & 0 & 4 & 24,9 & 22,2 & 7,5 & $\mathbf{5 8 , 7}$ \\
\hline País Vasco & 15 & 6 & 26,9 & 26,9 & 9 & $\mathbf{8 5 , 8}$ \\
\hline Murcia & 15 & 7 & 26,5 & 22,2 & 12 & $\mathbf{8 2 , 7}$ \\
\hline Media & 9,5 & $\mathbf{7 , 0}$ & $\mathbf{2 3 , 9}$ & $\mathbf{2 2 , 2}$ & $\mathbf{8 , 2}$ & $\mathbf{7 1 , 1}$ \\
\hline
\end{tabular}

Fuente: Elaboración propia. 
En concreto, los problemas detectados en lo que concierne a la buscabilidad están relacionados con la confusión que en algunos casos crea la diversidad de vías de ingreso en las webs autonómicas y el hecho, especialmente visible en las comunidades autónomas con menor puntuación, de que su posición en motores de búsqueda no resulte en todos los casos destacada.

En lo que atañe a la identidad, el estudio revela, prácticamente, la inexistencia de problemas: se informa adecuadamente de la entidad que respalda el portal, y una vez en la página correspondiente, el empleo de logotipos y tagline no suscitan dudas al usuario sobre el sitio web en el que se encuentra. Las comunidades más deficitarias al respecto muestran debilidades en la desaparición del logo identificador en páginas secundarias o, incluso, en la ausencia de datos que familiaricen (etiqueta "acerca de") al usuario con la actividad de la entidad que patrocina la página.

Los resultados relacionados con el diseño de las webs son, en general, positivos, pero no ocultan la presencia de anomalías importantes en algunos casos: diseños con escaso atractivo, empleo de etiquetas poco descriptivas o ambiguas -sirva de ejemplo el uso de la etiqueta "instrucciones previas" para aludir al acceso a los registros de voluntades anticipadas-, oferta inexistente o defectuosa de catálogos de servicios on line, presentaciones de contenidos no perfilados en función del tipo de usuarios, uso inadecuado del tamaño de los textos o falta de dispositivos multimedia asociados.

En la misma línea, los datos obtenidos sobre navegabilidad ofrecen, en general, una impresión favorable. Las deficiencias más graves detectadas atañen al mal funcionamiento de los dispositivos de elección de idioma (imposibilidad generalizada de acceder a los contenidos en, al menos, una lengua extranjera; a la alteración de la estructura de contenidos al cambiar la elección entre lenguas cooficiales); a la ausencia o mal funcionamiento de los motores de búsqueda propios o a la imposibilidad de acceder de forma rápida a los contenidos más buscados.

Por último, los datos relacionados con la seguridad revelan, nuevamente, una impresión tolerable, aunque cabe poner en evidencia la injustificable inexistencia de mecanismos de interacción mediante certificado digital o e-DNI en las comunidades autónomas que recibieron menor puntuación.

\section{Comunicación}

Al valorar la comunicación desde la perspectiva de test de experto se comprobó la disponibilidad de nueve dispositivos: asistente virtual, servicio de correo electrónico ofrecido por la propia entidad, dirección de correo electrónico de contacto, buzón virtual, repositorio de sugerencias y/o reclamaciones on line, medios de captación de opiniones de los usuarios (vía encuesta), foros de debate y consulta y, finalmente,

104 presencia activa del organismo en redes sociales. 
Los resultados obtenidos indican que el déficit de prestaciones ligadas a los mecanismos de comunicación es alarmante (tabla 3).

TABLA 3

Calidad de prestaciones relacionadas con los dispositivos de comunicación ofrecidos por las webs autonómicas de salud

\begin{tabular}{|c|c|c|c|c|}
\hline & $\begin{array}{c}\text { Atención } \\
(40 \%)\end{array}$ & $\begin{array}{c}\text { Consulta y } \\
\text { debate }(30 \%)\end{array}$ & $\begin{array}{c}\text { Sugerencias y } \\
\text { reclamaciones } \\
(30 \%)\end{array}$ & $\begin{array}{c}\text { TOTAL } \\
\text { (Sobre 100) }\end{array}$ \\
\hline Andalucía & 24 & 0 & 30 & 54 \\
\hline Aragón & 12 & 0 & 0 & 12 \\
\hline Asturias & 32 & 0 & 15 & 47 \\
\hline Baleares & 4 & 0 & 30 & 34 \\
\hline Canarias & 4 & 0 & 30 & 34 \\
\hline Cantabria & 4 & 0 & 0 & 4 \\
\hline $\begin{array}{l}\text { Castilla-La } \\
\text { Mancha }\end{array}$ & 0 & 0 & 30 & 30 \\
\hline Castilla y León & 24 & 15 & 30 & 69 \\
\hline Cataluña & 16 & 15 & 30 & 61 \\
\hline Madrid & 20 & 0 & 30 & 50 \\
\hline Valencia & 4 & 0 & 0 & 4 \\
\hline Extremadura & 24 & 15 & 0 & 39 \\
\hline Galicia & 12 & 15 & 30 & 57 \\
\hline La Rioja & 24 & 0 & 30 & 54 \\
\hline Murcia & 12 & 0 & 30 & 42 \\
\hline Navarra & 28 & 0 & 0 & 28 \\
\hline País Vasco & 20 & 0 & 30 & 50 \\
\hline Media & 15,5 & 3,5 & 20,2 & 39,3 \\
\hline
\end{tabular}

Fuente: Elaboración propia.

En particular, en lo que atañe a los dispositivos de atención al usuario (tabla 4), aunque se constata la generalización del buzón virtual como principal vía de contacto con la Administración y la disponibilidad de mecanismos para la formulación de reclamaciones, la lista de irregularidades detectadas es extensa:

- Funcionamiento defectuoso de los asistentes virtuales: operan de facto como repositorios no interactivos de "preguntas frecuentes" o motores de búsqueda propios, pero nunca como dispositivos de mensajería instantánea. 
- Remisión de las consultas de los usuarios a buzones virtuales "únicos", no especializados sectorialmente, para toda la Administración.

- Reducción de los mecanismos de participación a foros centralizados para toda la Administración, no especializados sectorialmente.

- Inexistencia o ubicación dificultosa de indicaciones de direcciones electrónicas de contacto en la página principal.

- Oferta de direcciones presuntamente orientadas a la atención sectorial al usuario (temas de salud) con destino a webmaster.

- Imposibilidad de acceder a redes sociales (allí donde permanecen activas) desde la página del portal.

— Desatención de cuentas en redes sociales.

- Remisión de las consultas en redes sociales a cuentas no especializadas sectorialmente.

- Alusión a mecanismos de participación on line no disponibles.

- Incapacidad de realizar sugerencias on line (necesidad de descargar formato en "pdf" y continuación de trámite off line).

— Ausencia de indicaciones de la dirección de e-mail de contacto.

Los mecanismos asociados a un enfoque de prestación de servicios y atención a los usuarios planteado desde la perspectiva de participación on line, en suma, próximos a las aspiraciones de las propuestas del e-gobierno 2.0, resultan deficitarios o infraadministrados: solo una Comunidad Autónoma recaba regularmente opiniones de los usuarios, tres mantienen activados foros de debate en relación con temas de salud, once permiten al usuario realizar sugerencias y solo seis disponen y nutren adecuadamente sus cuentas respectivas en redes sociales (tabla 4).

Son varias las webs autonómicas de salud que anuncian una próxima oferta de mecanismos de expresión de opiniones, expectativas, necesidades y sugerencias, así como dispositivos de captación de información y participación a través de foros y encuestas o, incluso, la apertura de nuevos canales interactivos de intercambio de experiencias que permitan la mejora de los servicios de salud, potencien el papel de los usuarios y las asociaciones en el diseño de la política sanitaria y posibiliten la intervención de la ciudadanía en la resolución de sus problemas de salud, complementando la acción de los servicios sanitarios. No obstante, por el momento, el nivel de de106 bajo. sarrollo de dispositivos adecuados de comunicación y participación on line es muy 
TABLA 4

Disponibilidad de medios de atención (on line) al usuario y estímulo de su participación ofrecidos por los portales autonómicos de salud

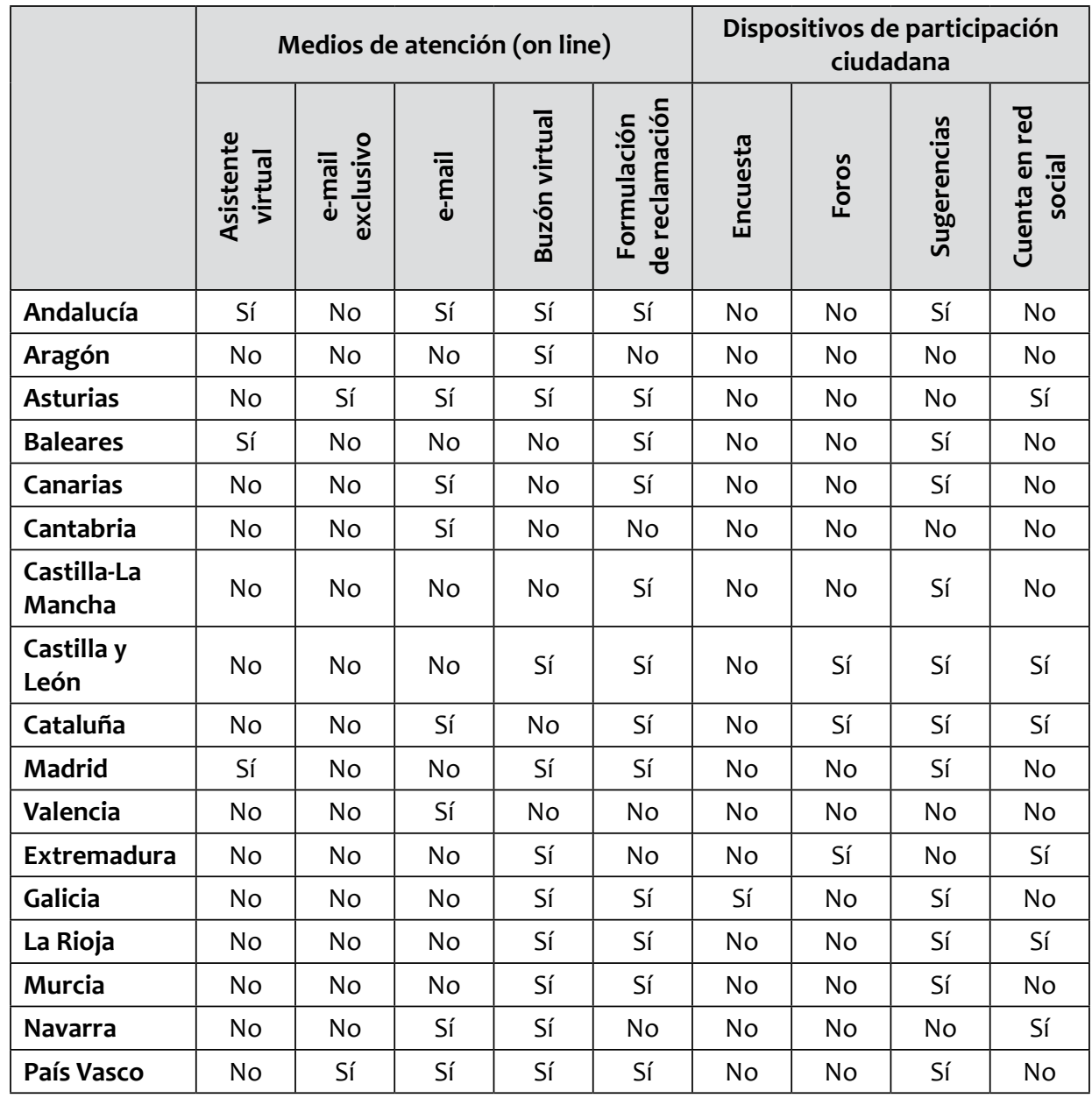

Fuente: Elaboración propia.

\section{Atención (vía web) al usuario}

Únicamente 9 administraciones ${ }^{4}$ han respondido a alguna de las demandas formuladas a través del experimento de e-usuario misterioso (tabla 5), siendo la de Aragón la única que responde a las cinco consultas planteadas.

4 Al no recibir respuesta a las consultas planteadas, no se incluyen en la tabla 5 las siguientes comunidades autónomas: Asturias, Canarias, Cantabria, Cataluña, La Rioja, País Vasco y Murcia. 
De la batería de requerimientos de información propuestos, la consulta relacionada con la tramitación de cita previa a través de Internet es la más atendida por los gestores de las webs autonómicas, pero ni siquiera por la totalidad de las comunidades autónomas que responden; con una frecuencia aún menor, las respuestas sobre las peticiones de información sobre un tema general de salud y sobre un procedimiento off line componen un segundo perfil de respuesta; finalmente, las consultas sobre la posibilidad de acceso a la historia clínica, en caso de desplazamiento a otra comunidad autónoma, y sobre el modo de realizar una sugerencia sobre el funcionamiento del sistema de salud son satisfechas solo por cinco administraciones autonómicas.

\section{TABLA 5}

Calidad de atención (vía web) al usuario en las páginas web de salud de las administraciones autonómicas 5

\begin{tabular}{|c|c|c|c|c|c|c|c|}
\hline 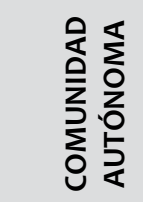 & 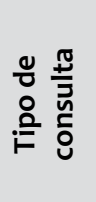 & 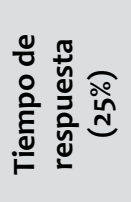 & 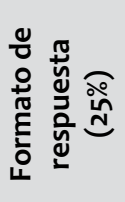 & 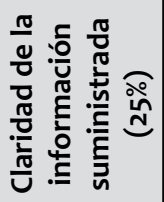 & 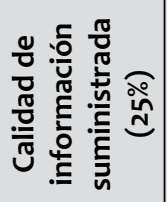 & 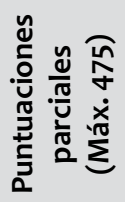 & 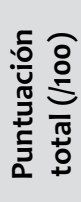 \\
\hline \multirow{5}{*}{ Andalucía } & $\mathrm{C}_{1}$ & 25 & 25 & 25 & 25 & 100 & \multirow{5}{*}{65,2} \\
\hline & $\mathrm{C} 2$ & 25 & 15 & 25 & 25 & 90 & \\
\hline & $C_{3}$ & NC & NC & NC & NC & NC & \\
\hline & $\mathrm{C}_{4}$ & 20 & 25 & 0 & 0 & 45 & \\
\hline & $C_{5}$ & 25 & 25 & 25 & NP & 75 & \\
\hline \multirow{5}{*}{ Aragón } & $\mathrm{C}_{1}$ & 20 & 5 & 15 & 25 & 65 & \multirow{5}{*}{61,0} \\
\hline & $\mathrm{C} 2$ & 20 & 15 & 25 & 25 & 85 & \\
\hline & $C_{3}$ & 20 & 5 & 5 & 5 & 35 & \\
\hline & $\mathrm{C}_{4}$ & 20 & 15 & 15 & 15 & 65 & \\
\hline & $C_{5}$ & 20 & 5 & 15 & NP & 40 & \\
\hline \multirow{5}{*}{ Baleares } & $\mathrm{C}_{1}$ & 25 & 25 & 15 & 5 & 70 & \multirow{5}{*}{72,6} \\
\hline & $\mathrm{C} 2$ & 25 & 25 & 25 & 25 & 100 & \\
\hline & $C_{3}$ & 25 & 25 & 25 & 25 & 100 & \\
\hline & $\mathrm{C}_{4}$ & NC & NC & NC & NC & NC & \\
\hline & $C_{5}$ & 25 & 25 & 25 & NP & 75 & \\
\hline
\end{tabular}

5 Tipos de consulta planteada: C1, Precauciones a adoptar en desplazamientos al extranjero; C2, Petición via web de cita en atención primaria; $\mathrm{C}_{3}$, Acceso a historia clínica; $\mathrm{C}_{4}$, Consentimiento informado; $\mathrm{C}_{5}$, Procedimiento para formular sugerencias; NC: No contesta; NP: No procede. 


\begin{tabular}{|c|c|c|c|c|c|c|c|}
\hline 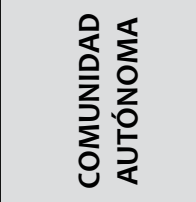 & 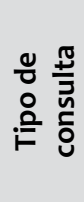 & 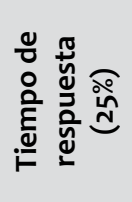 & 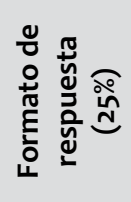 & 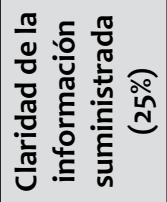 & 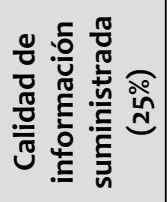 & 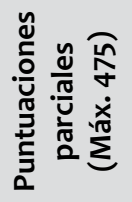 & 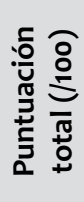 \\
\hline \multirow{5}{*}{$\begin{array}{l}\text { Castilla-La } \\
\text { Mancha }\end{array}$} & $C_{1}$ & $\mathrm{NC}$ & $\mathrm{NC}$ & $\mathrm{NC}$ & NC & NC & \multirow{5}{*}{29,4} \\
\hline & $\mathrm{C}_{2}$ & $\mathrm{NC}$ & $\mathrm{NC}$ & $\mathrm{NC}$ & $\mathrm{NC}$ & NC & \\
\hline & $\mathrm{C}_{3}$ & 20 & 25 & 25 & 25 & 95 & \\
\hline & $\mathrm{C}_{4}$ & $\mathrm{NC}$ & $\mathrm{NC}$ & $\mathrm{NC}$ & $\mathrm{NC}$ & NC & \\
\hline & $C_{5}$ & 25 & 5 & 15 & NP & 45 & \\
\hline \multirow{5}{*}{$\begin{array}{l}\text { Castilla y } \\
\text { León }\end{array}$} & $\mathrm{C}_{1}$ & $\mathrm{NC}$ & $\mathrm{NC}$ & $\mathrm{NC}$ & $\mathrm{NC}$ & NC & \multirow{5}{*}{37,8} \\
\hline & $\mathrm{C} 2$ & 15 & 15 & 15 & 15 & 60 & \\
\hline & $\mathrm{C}_{3}$ & 15 & 15 & 15 & 15 & 60 & \\
\hline & $\mathrm{C}_{4}$ & 15 & 15 & 15 & 15 & 60 & \\
\hline & $\mathrm{C}_{5}$ & $\mathrm{NC}$ & $\mathrm{NC}$ & $\mathrm{NC}$ & NP & NC & \\
\hline \multirow{5}{*}{ Madrid } & $\mathrm{C} 1$ & 25 & 25 & 25 & 25 & 100 & \multirow{5}{*}{57,8} \\
\hline & $\mathrm{C} 2$ & 25 & 25 & 25 & 25 & 100 & \\
\hline & $\mathrm{C}_{3}$ & $\mathrm{NC}$ & $\mathrm{NC}$ & $\mathrm{NC}$ & $\mathrm{NC}$ & NC & \\
\hline & $\mathrm{C}_{4}$ & 20 & 25 & 15 & 15 & 75 & \\
\hline & $\mathrm{C}_{5}$ & $\mathrm{NC}$ & $\mathrm{NC}$ & $\mathrm{NC}$ & NP & NC & \\
\hline \multirow{5}{*}{ Valencia } & $\mathrm{C} 1$ & 20 & 5 & 15 & 5 & 45 & \multirow{5}{*}{49,4} \\
\hline & $\mathrm{C} 2$ & 20 & 15 & 15 & 15 & 65 & \\
\hline & $\mathrm{C}_{3}$ & 20 & 15 & 15 & 15 & 65 & \\
\hline & $\mathrm{C}_{4}$ & 15 & 15 & 15 & 15 & 60 & \\
\hline & $\mathrm{C}_{5}$ & $\mathrm{NC}$ & $\mathrm{NC}$ & $\mathrm{NC}$ & $\mathrm{NP}$ & NC & \\
\hline \multirow{5}{*}{ Extremadura } & $\mathrm{C} 1$ & $\mathrm{NC}$ & $\mathrm{NC}$ & $\mathrm{NC}$ & NP & NC & \multirow{5}{*}{11,5} \\
\hline & $\mathrm{C} 2$ & 25 & 15 & 15 & 0 & 55 & \\
\hline & $C_{3}$ & $\mathrm{NC}$ & $\mathrm{NC}$ & $\mathrm{NC}$ & $\mathrm{NC}$ & NC & \\
\hline & $\mathrm{C}_{4}$ & $\mathrm{NC}$ & $\mathrm{NC}$ & $\mathrm{NC}$ & $\mathrm{NC}$ & NC & \\
\hline & $C_{5}$ & $\mathrm{NC}$ & $\mathrm{NC}$ & $\mathrm{NC}$ & $\mathrm{NP}$ & NC & \\
\hline \multirow{5}{*}{ Galicia } & $\mathrm{C} 1$ & 20 & 25 & 25 & 25 & 95 & \multirow{5}{*}{73,6} \\
\hline & $\mathrm{C}_{2}$ & 25 & 25 & 25 & 25 & 100 & \\
\hline & $C_{3}$ & $\mathrm{NC}$ & $\mathrm{NC}$ & $\mathrm{NC}$ & $\mathrm{NC}$ & NC & \\
\hline & $\mathrm{C}_{4}$ & 15 & 25 & 25 & 25 & 90 & \\
\hline & $C_{5}$ & 15 & 25 & 25 & NP & 65 & \\
\hline \multirow{5}{*}{ Navarra } & $\mathrm{C}_{1}$ & 15 & 15 & 15 & 15 & 60 & \multirow{5}{*}{45,2} \\
\hline & $\mathrm{C}_{2}$ & 15 & 25 & 25 & 25 & 90 & \\
\hline & $C_{3}$ & $\mathrm{NC}$ & $\mathrm{NC}$ & $\mathrm{NC}$ & $\mathrm{NC}$ & NC & \\
\hline & $\mathrm{C}_{4}$ & 10 & 25 & 15 & 15 & 65 & \\
\hline & $C_{5}$ & $\mathrm{NC}$ & $\mathrm{NC}$ & $\mathrm{NC}$ & NP & NC & \\
\hline
\end{tabular}

Fuente: Elaboración propia. 
La receptividad pobre de los gestores de servicios públicos autonómicos de salud a las consultas realizadas vía web es compensada por el logro de una calidad de respuesta aceptable-oscila entre los 60 y 82 puntos porcentuales en una escala en la que la proximidad a 100 connotaría los niveles máximos de calidad--correspondiente a un patrón de atención en el que la respuesta al usuario se produce entre las 36 y 48 horas tras efectuarse la consulta, mediante el empleo de un formato bien estructurado $(7,3$ puntos sobre 10) y comprensible (7,4 puntos sobre 10), aunque no completamente ajustado (6,9 puntos sobre 10) a la temática planteada, ni abundante en lo relativo a la facilitación de información complementaria así como a datos de referencia y contacto que, eventualmente, permitan al usuario proseguir su búsqueda de información.

\section{TABLA 6}

Resultados generales del examen a las prestaciones de usabilidad, dispositivos de comunicación y calidad de atención al usuario en los portales autonómicos de salud ${ }^{6}$

\begin{tabular}{|c|c|c|c|c|c|c|}
\hline & $\begin{array}{c}\text { A } \\
\text { Usabilidad } \\
(/ 100)\end{array}$ & $\begin{array}{c}\text { B } \\
\text { Comunicación } \\
(/ 100)\end{array}$ & $\begin{array}{c}\text { C } \\
\text { Atención al } \\
\text { usuario } \\
(/ 100)\end{array}$ & $\begin{array}{c}\text { Total } \\
A+B \\
(/ 200)\end{array}$ & $\begin{array}{c}\text { Total } \\
A+B+C \\
(/ 300)\end{array}$ & $\begin{array}{l}\text { Total } \\
(/ 10)\end{array}$ \\
\hline Andalucía & 77,0 & 54 & 65,2 & 131,0 & 196,3 & 6,5 \\
\hline Aragón & 58,4 & 12 & 61,0 & 70,4 & 131,4 & 4,3 \\
\hline Asturias & 66,9 & 47 & 0 & 113,9 & 113,9 & 3,7 \\
\hline Baleares & 62,3 & 34 & 72,6 & 96,3 & 168,9 & 5,6 \\
\hline Canarias & 64,4 & 34 & 0 & 98,4 & 98,4 & 3,2 \\
\hline Cantabria & 58,6 & 4 & 0 & 62,6 & 62,6 & 2,0 \\
\hline $\begin{array}{l}\text { Castilla-La } \\
\text { Mancha }\end{array}$ & 74,6 & 30 & 29,4 & 104,6 & 134,0 & 4,4 \\
\hline Castilla y León & 63,5 & 69 & 37,8 & 132,5 & 170,4 & 5,6 \\
\hline Cataluña & 69,0 & 61 & 0 & 130,0 & 130,0 & 4,3 \\
\hline Madrid & 82,6 & 50 & 57,8 & 132,6 & 190,5 & 6,3 \\
\hline Valencia & 64,0 & 4 & 49,4 & 68,0 & 117,5 & 3,9 \\
\hline Extremadura & 74,1 & 39 & 11,5 & 113,1 & 124,6 & 4,1 \\
\hline Galicia & 79,5 & 57 & 73,6 & 136,5 & 210,2 & 7,0 \\
\hline La Rioja & 86,8 & 54 & 0 & 140,0 & 140,0 & 4,6 \\
\hline Navarra & 58,7 & 28 & 45,2 & 86,7 & 131,9 & 4,4 \\
\hline País Vasco & 85,8 & 50 & 0 & 135,8 & 135,8 & 4,5 \\
\hline Murcia & 82,7 & 42 & 0 & 124,7 & 124,7 & 4,1 \\
\hline Media & 71,1 & 39,3 & 29,6 & 103,1 & 110,4 & 4,6 \\
\hline
\end{tabular}

Fuente: Elaboración propia.

6 En negrita: resultados que igualan o superan el 50\% de la puntuación máxima concedida por 


\section{Resultados generales}

Partiendo del supuesto de la concesión de un peso similar a las diversas dimensiones examinadas (usabilidad, dispositivos de comunicación y atención al usuario), el examen efectuado a las webs gestionadas por las administraciones autonómicas demuestra que las utilidades y elementos infraestructurales dependientes directamente de la inversión en tecnología y, en concreto, los componentes relacionados con la usabilidad (visibilidad de la página y facilidad de detección a través de motores de búsqueda, identidad, diseño, navegabilidad y seguridad) han gozado de un desarrollo mayor que el de los dispositivos de comunicación y, sobre todo -a juzgar por su rendimiento-, que el de los mecanismos de atención al usuario: la media de las funcionalidades ligadas a la usabilidad alcanza una puntuación de 71,14 sobre una escala de 100 puntos máximos posibles, superando el $50 \%$ en todas las comunidades autónomas; la puntuación media alcanzada por los dispositivos de comunicación desciende a 39,35 (sobre 100) y solo superan la barrera del 50\% siete administraciones (Andalucía, Castilla y León, Cataluña, Madrid, Galicia, La Rioja y País Vasco); mientras que la puntuación media lograda por las comunidades autónomas en lo concerniente a la atención al usuario solo alcanza 29,65 puntos (sobre 100), siendo tan solo superado el $50 \%$ de la puntuación máxima posible en 5 territorios (Andalucía, Aragón, Baleares, Madrid y Galicia).

Para remarcar la comprensibilidad de los datos resultantes, se ha incorporado a la tabla 6 una columna cuyas valoraciones totales se sitúan en una escala de 0 a 10, en la que se considera aceptable todo resultado igual o superior a 5 , un umbral solo superado por las webs de salud de cinco comunidades autónomas (Andalucía, Baleares, Castilla y León, Madrid y Galicia), que la media de la totalidad de administraciones autonómicas no alcanza.

\section{CONCLUSIONES}

Este trabajo ha presentado los resultados de un estudio sobre los portales web que las administraciones autonómicas ofrecen en el área de salud y, en concreto, sobre la disponibilidad y capacidad de los dispositivos de comunicación ofrecidos.

Los objetivos que han guiado la investigación han sido identificar la presencia de dispositivos facilitadores de la comunicación bidireccional vía web, examinar su grado de desarrollo y verificar su capacidad real para suscitar interactividad y, en consecuencia, contribuir a satisfacer las demandas de los usuarios. Para ello se emplearon de forma combinada dos técnicas: test de experto de usabilidad técnica y comunicación y experimento de e-usuario misterioso.

Los principales hallazgos derivados de la aplicación del test heurístico revelan, por una parte, que, aunque las prestaciones relacionadas con la usabilidad técnica de las webs autonómicas de salud son, en general, adecuadas y, por tanto, no suscitan 
obstáculos de relieve - derivados de ubicuidad, identidad, diseño, navegabilidad y seguridad- que impidan el establecimiento de una comunicación interactiva on line entre Administración y usuarios, el déficit de mecanismos que le dan soporte es evidente, resultando especialmente grave la ausencia de dispositivos adecuados de atención a los usuarios y de aplicaciones generadoras de consulta y debate propios de un entorno de participación 2.0.

Por otra parte, como resultado de la realización del experimento de e-usuario misterioso, si bien el nivel de respuesta de las comunidades autónomas es bajo, la calidad de las mismas se sitúa en un nivel medio.

\section{REFERENCIAS BIBLIOGRÁFICAS Y DOCUMENTALES}

Andreassen, H.K. et al. (2007), “European citizens' use of E-health services: A study of seven countries", BMC Public Health, 7:53.

AT Internet (2011), “Barómetro de buscadores, febrero 2011”. En línea: <http://es.atinternet.com/Recursos/Estudios/Barometro-buscadores/Search-Engine-Barometer-January-2011/index-1-2-6-223.aspx> (consulta: 23 febrero 2011).

Comisión Europea (2002), eEurope 2005: An information society for all. European Commission-COM (2002) 263 final. En línea: < http://ec.europa.eu/information_society/eeurope/2002/news_library/documents/eeurope2005/eeurope2005_en. pdf> (consulta: 23 julio 2011).

Comisión Europea (2004), e-Health-Making healthcare better for European citizens: An action plan for a European e-Health Area. COM (2004) 356.

Dumas, J.S. y Redish. J.C. (1999), A Practical Guide to Usability Testing. Bristol: Intellect.

Eysenbach, G. (2008), “Medicine 2.0: Social Networking, Collaboration, Participation, Apomediation, and Openness", Journal of Medical Internet Research, 10 (3):e22.

Hassan, Y. y Martín, F.J. (2003), "Guía de Evaluación Heurística de Sitios Web". No Solo Usabilidad. Revista multidisciplinar sobre diseño, personas y ciudadanía, 2. En línea: <http://www.nosolousabilidad.com/articulos/heuristica.htm> (consulta: 23 julio 2011).

Kruk, S. (2000), Don't Make me Think! A Common Sense Approach to Web Usability. Indianapolis: Que Coorp.

Kruk, S. (2001), No me Hagas Pensar: Una Aproximación a la Usabilidad en la Web. Madrid: Pearson.

Leuenberger, R., Schegg, R. y Murphy, J. (2003), "E-mail customer service by upscale international hotels”, en A.J. Frew, M. Hitz y P. O'Connor, eds., Information and Communication Technologies in Tourism. Nueva York: Springer.

Matzler, K., Pechlaner, H., Abfalter, D. y Wolf, M. (2005), "Determinants of response to customer e-mail enquiries to hotels: evidence from Austria", Tourism Management, 26: 249-259.

Nielsen, J. (1994), “Heuristic Evaluation”, en J. Nielsen y R.L. Mack, eds., Usability In112 spection Methods. Nueva York: John Wiley \& Sons. 
OCDE (2005), E-Government for Better Government. Paris: OECD E-Government Studies.

OMS/WHO (2011), Atlas eHealth Country Profiles 2010. Ginebra: WHO.

Schedler, K. y Proeller, I. (2003), New Public Management. Berna: Paul Haupt.

UNDESA (2010), E-Government Survey 2010. Leveraging e-government at a time of financial and economic crisis. Nueva York: Naciones Unidas.

Voss, C. (2000), "Developing an e-service strategy”, Business Strategy Review, 11(1), 21-33. 
